\title{
PERFIL DO PORTADOR DE LESÃO CRÔNICA DE PELE: FUNDAMENTANDO A AUTOPERCEPÇÃO DE QUALIDADE DE VIDA
}

Profile of the carrier of chronic injury of skin:

basing the self-perception about quality of life

Perfil del portador de lesión crónica de la piel:

fundamentando la autopercepción de calidad de vida

AraludiaPazos Dias

Lolita DopicodaSiva

\section{Resumo}

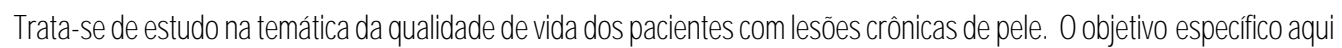

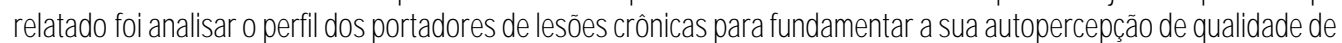
vida Estudoprospectivo, dbsenvaiond, commoddo deinten enção eanálisequantitativa. Foi adicado oquestionário WHOQOL-bref noperíodo deseis meses a una população compostade 40 indvíduos quefreqüentaramduranteseis

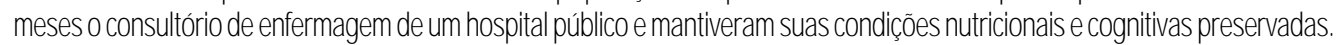
Os resultadbs nostramque 75\% sãodientes ainæade51 anos; 55\%sãohonens, 70\%vivemsozinhos, 52,5\%têmo primerirodidodbensinofundanertal; $6,5 \%$ nãotrabalhame 32,5\% nãotêmqualquertipoderenda, e90\%possuemTV

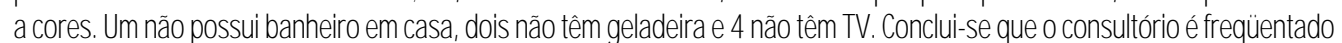
por pessoas comlimitações financeiras. Isso reforçaaimportânaiadeseverificar a autoperceppãão dequalidadedevida apertir dacompreensão dos determinartes socioeconônicos dogn poestudadb.

Palaras-dhave Efermagem Qualidadede Vida DoençaCrônica

\begin{abstract}
Resumen

This is a study about the quality of life of the petients with Estees unestudiosobrelacalidaddelavidadelospadentes dronicinivies of skin Thespecific dijective ves to andyze conlesiones crónicas dela pie. El objetivo espećfico era theprofile of the camiers of droniciniuries to basethem analizar e perfil delos partadores delesiones crónicas para self-perception about quality of life. Prospective, basarlas unauto-percepcón sobrelacalidaddelavica. dbsentiond study, withmodl of intenertionandquantitative Estudio anticipadb, de obsenación, con el modelo de la andysis. TheWHOOQ-bref questionaireves apdiedinthe intenencón y del análisis a antitativo. E arestionario de period of six months to a composed population of 40 WHOOQ-breffieaplicadbene periodbdeseismesesauna

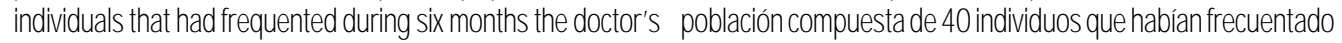
office of nursing of a public hospital and had kept its durante seis meses $\mathrm{I}$ consuttorio de la enfermera de un preserved nutritiond and cogritiveconditions. Theresuts hospital públicoy resguerdandbsuscondiones dimerticias vere: $75 \%$ areastomers above 51 years; $55 \%$ aremen, ycognosatives. Los resultadbs fueron $75 \%$ sondientes

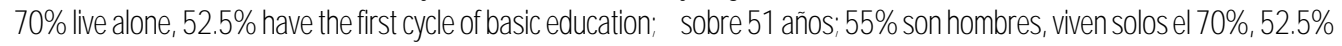
$67.5 \%$ do not nork and $32.5 \%$ do not have any type of tienene primercidodelaed radónbásica $6.5 \%$ motrabajan incomeand $90 \%$ possess color TV. Onedbes not possess y32.5\% mohacen a dquier tipoderentay $90 \%$ poseen TV. battroominthehouse, two do notheverefrigerator and 4 Unonoposeebañoencasa, dos notienen refrigeradory 4 donothaveanykind of TV. Itwes condudethat thedbator's motienenning madasedeTV. Seconduyequed consultorio officeis frequerted by peqplevith finandial limitations. That es freartado por gentecon limitaciones finanderas. Eso strengthens theimportance of to verifytheself-perception consolidalaimportaniadeverificarlaquinónsdbrecalicad about quality of life from the understanding of the de vida desde la comprensión de los determinartes determinativesocioeconomics of thestudedgrap. socioeconónicos del grupoestudiado.
\end{abstract}

Keymords
Nusing Qulity of Life CronicDiseese

\section{Palchasdae:}

Efermería CalidaddeVica. ErfermedadCrónica. 
INTRODUÇÃO

Este artigo refere-se a umdos resultados parciais de uma dissertação de mestrado desenvolvida e aprovada no Programa de Mestrado da Faculdade de Effermagemda U⿴囗十. Tratase de umestudo centrado na ternática da qualidade de vida dos pacientes com lesões crônicas de pele que são atendidos emum consultório espedializado de Enfermagem em um hospital público. Atualmente, as condições crônicas são responsáveis por $60 \%$ de todo o ônus financeiro decorrente de dbenças do mundo, e, no ano de 2020, $80 \%$ da carga de doença dos países em desenvolvimento devemadir de problemas crônicos'. Isso faz pensar na necessidade de nodificar as metas assistenciais de todb hospital público que se restringem a tratar o paciente na manifestação aguda de sua doença de forma estanque e não resolutiva. $\mathrm{Na}$ verdade, não há dsporibilidade suficiente nes Unidades de Saúde para atenção a condições crônicas em especial ao tratamento de feridas crônicas?.

Nesse contexto estão os pacientes portadores de lesões crônicas de pele, e os nesmos acabamtentando soluções próprias, baseadas emaidados empíicos, mitas vezes por recomendações de amigos ou familiares, ocasionando abordagens terapêuticas inadequadas, insegurança e desgaste à sua saúde. Portanto, ser portador de una doença crônica sempre afeta a percepgãa de qualidade de vida.

A expressão qualidade de vida temsido utilizada comdiversos enfoques: na área política como citério de racioánio, visando identificar as condiçōes de vida populacionais, e, mais consistentemente, na literatura científica, partilhada por cientistas sociais e filósofos, originalmente, onde parece surgir a maioria dos esforços para dimensionar qualidade de vida. Hoje, qualidade de vida é terma de pesquisas impresandíveis na área da saúde, visto que seus resultados contribuem para aprovar e definir tratamentos e avaliar o asto/ benefício do aidado prestado. Una importante organização intemacional que desemperha umpapd decisivo no incentivo e estudos para a qualidade de vica é a Organização Mndial de Saúde (OMS) que em 1948, definiu a saúde como umcompleto estado de bemestar físico, mental e social, e não somente ausência de enfermidade

Atudmerte, a saúde de uma pessoa avaliase além de sua capacidade física, levando-se emconta seu contexo social e sua saúde menta. Esta idéa ao longo do tempo evoluiu pera o conceito norteador do estudo realizado pelo Gupo de qualidade de vida da OMS que pessou a definir qualidade de vida comr: a perceppção do irdvído arerca de sua posição ra vida, no conteto da cltura e sistenæs de valores nos quais de vive e emrelação aos sess djietios, expectatives, padrões e preoupaçõe $e^{3: 3}$

Assim qualidade de vida é um construto que compreende mútiplas dinensões da vida humana Para o grupo WHOOOL estas dimensões são: a dimensão física, a psicológica, o nível de independência, as relações sociais e o meio ambiente Neste estudo, optourse pela dimensão física por entendêla com sendo, provavelmente, aquela percebida mais significativamente pelos enfermeiros nas unidades hospitalares e tambémnas pessoas comalgumagravo à saúde, já que incorpora qualquer aspecto que pertença ao seu corpo e à sua funcionalidade.

O problema da pesquisa do estudb consistiu em saber qual é a variação que se obsena no dońriofísicodaqua dededevidadepacientes comlesöes aûicas de pele qandb alendidos em um consuthório de enfermagem? Essa variação foi avaliada aplicando-se aos pacientes portadores de lesões crônicas de pele o questionário do grupo WHOSOL, o "WHOQOL-bref", que consta de 26 questões, sendo duas questões gerais de qualidade de vida, e as demais 24 representamcada uma das 24 facetas que compõem o instrumento original (WHOOa 100) ${ }^{34}$.

A pesquisa teve vánios objetivos espećficos, entre eles analisar o perfil dos pacientes portadores de lesão crônica de pele e que freqüentam umconsultório de enfermagem de hospital público. O grupo WHOOOL orienta que qualquer pesquisa que envolva a autopercepção de qualidade de vida deve vir precedida do levantamento do perfil dos sujeitos que estão opinando sobre sua qualidade de vida3-4.

\section{METODOLOGIA}

Tratourse de umestudb prospedivo, obsenvecional, com modelo de intenvenção e análise de dados quantitativa. A pesquisa foi autorizada emdezentbo de 2004 pelo Conitê de Ética do próprio hospital. O estudo foi realizado comos padientes que freqüentam umconsultónio de enfermagem de lesões de pele de umhospital público geral da rede federa, o Hospital Geral de Bonsucesso, no muicápio do Rio de Janeiro. Consideraramse população-fonte os pacientes com lesões crônicas de pele acompanhados ambulatorialmente após alta hospitalar. Nb consultónio de enfermagem são atendidos, em média, 25 pacientes por dia, compondo uma agenda de 
aproximadamente 140 padientes comlesões crônicas e comlesões agudas. Selecionaramse então, para esse estudo, aquelas pessoas comlesões crônicas dentro dos seguintes citérios de indusão: a) ter lesão crônica de pele - a condição crônica impõe necessidades e co-morbicades que constituem fatores importantes na determinação da qualidade de vida; b) ter umaidador - como os padientes apresentam limitações para as suas atividades, a garantia da continuidade do tratamento no domílio é assegurada coma presença de umaidadbr, c) perticipar de todas as consultas - o seguimento regular das consultas possibilita o gerenciamento do aidado permitindb, dessa forma, avaliação contínua para identificar as dficuldades individuais, intenvindo imediatamente; d) ter cognitivo preservado - para assegurar o entendimento das orientações ministradas e também a compreensão do conteúdo do questionário ministrado; e) assinar o Termo de Consentinento Live e Esdaredido - nerhumpaciente se negou a assinar o terro. Os critérios de exdusão foram apresentar déficit nutricional, hemodinâmico e/ou neurológico e rão permanecer como aidador. Assim 40 padientes constitúrama população do estudo.

Estratégias para Construça dos Dados A pesquisa foi desenvolvida emtrês etapas. A primera consistiu na sistematização da consulta e do tratamento de feridas crônicas, o que demandou em tomo de sessenta dias. Nesse período foi elaborado umprotocolo para o tratamento de lesões crônicas de pele fundamentado em extensa pesquisa bibliográfica. Foi montada a infra-estrutura de um consultório de enfermagem após a Comissão de tratamento de fericlas ser instituída por portaria pela direção do hospital. A segunda etapa foi o desenvolvimento das consultas de enfermagemonde se aplicava o mestro protocolo de tratarento da ferica crônica a todbs os 40 pacientes que compunhama população do estudo, e, nesse monerto, se identificou o perfil dos sujeitos participartes do estudb. A terceira etapa foi a aplicação do questionánio sobre qualidade de vida a esses 40 padientes de 15 em 15 dias à nedida que eles recebiamo atendimento padronizadb no ambulatório de enfermagem Essa última etapa fai realizada emumperíodo de seis neses (dezentro de 2004 a maio de 2005), utilizando como instru mento de coleta de dadbs o questionário do gupo WHOOSA, o WHCOQ-bref, queconsta de 26 questões, sendo dies questões gerais de qualidade de vida, e as derris 24 representamcada u ma das 24 facetas que compõem o instrumento original (WHOSO 100). As respostas para todas as questões do WHOOQL-bref foramodtidas através de una escala de respostas do tipo likert com uma escala de intensidade (nada - extremamente), capacidade (nada - completamerte), frequiênaia (nunca - sempre) e avaliação (mito insatisfeito - mito satisfeito; mito nim- mito bom). Nelas, as palavasâncoras receberamo código 1 e 5, e as diferentes graduações entre estes dois extremos receberamo códgo 2, 3 e 4. Procedeurse, então, a identificação da correlação entre os escores das facetas que compõem o domínio físico através do teste da razão de verossimilhança entre os dados de UKERT na 1a aplicação e as subseqüentes, acompanhada de análise exploratónia das linhas de dados. Isto é, foi realizada a razão de verossimilhança entre a $1^{\mathrm{a}}$ e a $2^{\mathrm{a}}$ aplicação, seguida da razão entre a primeira e as sucessivas aplicações. O tempo transcorrido entre a primeira medida e a citava foi de aproximadanente 6 meses, e, durante este tempo, cada padiente recebeu, emmédia, 20 intervenções, isto é, foramsulumetidos ao tratamento de lesões de pele no consultónio de enfermagemem média 20 vezes. A pesquisa foi realizada de acordo comas resoluções do Conselho Nacional de Saúde de números 196 de autubro de 1996 e 251 de agosto de 1997. Os pacientes que participaram do estudo receberamexplicação verbal acerca dos procedimentos a seremrealizados e foramsolicitadbs a assiner o term de consertimento live e esdaredido.

\section{RESULTADOS E DISCUSSÃO}

A análise dos resultados do perfil da população é imprescinóviel nos estudos sobre qualidade de vida e tempor finalidade avaliar e ponderar a consistência dos escores referidos pelos pacientes sobre a sua qualidade de vida, bem como determinar a sua confiabilidade Desta forma, pode-se aumentar o alcance de compreensão dos resultados com conseqüente validação da pesquisa ${ }^{-7}$.

Os dados que compuseramo perfil dos sujeitos foram idade, sexo, estado conjugal, escolaridade, condção labora, bens de consumo.

Emrelação à idade, constatamos, na Tabela 1, una população composta de 22 homens e 18 milheres. Destes, 75\% estão acima dos 51 anos, sendo que predominam os homens idosos, e a minoria da população (7,5\%) está no grupo etário de 20 a 30 anos e 7,5\% entre 31 e 40 anos. Por ser umestudo que foca lesões que via de regra energemde patologias crônico-degenerativas é coerente o predomínio de 
idosos. Na relação entre longevidade e qualidade de vida, existemcombinações de váios fatores, entre eles dbenças bidógicas e mentais, controle cognitivo, perdas de papés ocupadionais e status social, competência social, produtividade, relações formais e informais com familiares e amigos e perdas afetivas. Qualidade de vida nessa idade implica, portanto, no entendimento de determinantes de bemestar que repousa tanto no campo físico quanto mental, relacionamentos interpessoais, espiritual e material'

Os dados na Tabela 1 tambémsugerem que a controvérsia que a literatura leventa de que é maior a susceptibilidade do sexo ferminimo às dbenças crônicas não encontra exemplo disponível neste estudo. Esta discussão baseia-se no fato de que, à medida que a expectativa de vida foi aumentando, aumentaram também as diferenças de idade entre homens e milheres, de tal forma que, emmédia, as milheres vivemánco anos mais que os homens. ${ }^{7}$

Tabela 1

Composição por idade e sexo da população- HG B-2005

\begin{tabular}{|c|c|c|c|c|c|c|}
\hline \multirow{2}{*}{$\begin{array}{l}\text { Gr.jpos de } \\
\text { idace } \\
\text { íllus }\end{array}$} & \multicolumn{2}{|c|}{ MHSELULINO } & \multicolumn{2}{|c|}{ EFIN|NO } & \multicolumn{2}{|c|}{ TOTAL } \\
\hline & $N_{j}$ & 5 & Ne: & $\%$ & $\mathrm{Nu}$ & 5 \\
\hline 30 & 02 & 5.0 & & 2.5 & 3 & 7.5 \\
\hline $3--40$ & 1 & 2,3 & $?$ & 50 & 3 & $7, \overline{3}$ \\
\hline$\angle \quad 59$ & $?$ & 5,0 & $?$ & 5,0 & 4 & 10,0 \\
\hline $4---139$ & $\hat{~}$ & Th, & h & 12,4 & 11 & 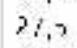 \\
\hline Mzis de 50 & 11 & 27,5 & $B$ & 20,0 & 13 & $47, \overline{3}$ \\
\hline TOTMLL & 22 & 55,0 & 18 & 45,0 & 40 & 130.0 \\
\hline
\end{tabular}

Emrelação à urião estável, dbservarse, ra Tabela 2, que $70 \%$ da população não mantêmnenhumtipo de união estável e que estes são exatamente os que estão adina de 51 anos. Neste sentido, desde 1983, o IBCE ${ }^{8}$ já vemrevelando uma grande proporção de pessoas idosas que vivemsós. Dentre as mitas questões da terceira idade, uma delas é o sentimento da solidão e exdusão social que se associa até aos aiidados com a saúde, com a higiene pessoal e domialiar ${ }^{9}$. Estes fatores permeiama qualidade de vida já que se trata de idosos que vivemsozinhos e predisamcriar condições que lhes garantamo methor equilíbrio possível nas condições emque vivem $O$ suporte familiar que traz o bemestar sodial é um importante aspedo da avaliação de qualidade de vica. Representa segurança e apoio para lidar com as mudanças temporárias ou permanentes da idade e da condição crônica ${ }^{9}$
Tabela 2

Composição por idade e estado conjugal da população. HG B-2005

\begin{tabular}{|c|c|c|c|c|c|c|}
\hline \multirow{2}{*}{$\begin{array}{c}\text { Gl dpus de } \\
\text { dade } \\
\text { iaros! }\end{array}$} & \multicolumn{2}{|c|}{$\begin{array}{l}\text { UI "av } \\
\text { estável }\end{array}$} & \multicolumn{2}{|c|}{ Sozintos } & \multicolumn{2}{|c|}{ TOTAL } \\
\hline & No & 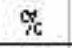 & $\mathrm{No}$ & 帒 & $\mathrm{No}_{\mathrm{O}}$ & $\pi$ \\
\hline $2^{-}-\cdots 30$ & 03 & $7, \overline{3}$ & 0 & 3 & 3 & $7 ! 5$ \\
\hline $3^{-\cdots-10}$ & 3 & 7,5 & 0 & 3 & 3 & 7,5 \\
\hline$\therefore-\ldots$ & 4 & 10,0 & 0 & 0 & 4 & 10.2 \\
\hline $5 \quad 60$ & ? & 2 & 11 & 27,5 & 11 & 77,5 \\
\hline Mzis de 60 & i) & ;) & 19 & $-4,5$ & 19 & $4 / .5$ \\
\hline TOTA & 10 & 30,0 & 30 & 70,0 & 40 & 100.0 \\
\hline
\end{tabular}

A escolaridade da população foi elencada pela importância deste dado no entendimento e prosseguimento das orientações recebidas durante 0 atendimento no consultório de enfermagem Neste estudo, o predomínio é de pessoas como primeiro segmento do ensino fundamental completo (52,5\%), seguido do ensino fundamental completo (15\%). Há três pessoas que se dedararamanalfabetas (7,5\%), e uma informou ter grau superior incompleto. Ofato de encontrar pessoas analfabetas não era esperado, por se tratar de umcentro urbano, complanejamento de ações educativas e inúmeras oportunidades de affabetização. Umdéficit na escolaridade pode interferir diretamente na continuidade do aidado no domiálio e no desenvolvimento da consciênda sanitária. Pode tambéminterferir no atendimento às prescrições de enfermageme na prática do autociidado, o que se desdobra em indicadores de qualidade de vida?

A condição laboral é demonstrada na Tabela 3. Aqui houve interesse de fazer uma dupla associação. Ao saber se trabalhavam procurourse saber em que trabalhavame se timhamúnalo empregatício. Os que trabalhamcorrespondiama 37,5\% Entre o grupo que não trabalhava, predominavamos que não tinham nerhumtipo de renda. Entre os que possúamrenda, predominava a renda da aposentadoria por invalidez.

\section{Tabela 3}

Distribuição da população de acordo com a condição laboralHG B-2005

\begin{tabular}{|c|c|c|c|c|c|c|}
\hline \multirow{2}{*}{$\begin{array}{l}\text { Gruacs de } \\
\text { idace } \\
\text { icullus? }\end{array}$} & \multicolumn{2}{|c|}{ Trataha } & \multicolumn{2}{|c|}{$\begin{array}{c}N=\sigma_{0} \\
\text { trabalra }\end{array}$} & \multicolumn{2}{|c|}{ TOTAL } \\
\hline & $\omega$ & $\%$ & 30 & $\%$ & $\mathrm{Nu}$ & 80 \\
\hline Péblico & 2 & 5 & $\mathrm{C}$ & 0 & 2 & 5 \\
\hline Priads & 4 & 10.0 & c & 0 & 4 & 10,0 \\
\hline Autônono & 6 & 15 & c & 0 & 6 & 13 \\
\hline ùfosen:ado & 0 & 0 & $\because$ & 35 & 11 & 35 \\
\hline Se'rl rarida & 0 & 9 & -3 & 32,5 & 13 & 32,5 \\
\hline Persäo & & & 1 & 7,5 & 1 & 7.5 \\
\hline TOThL & -2 & 30 & 28 & 67.5 & 40 & $100 \mathrm{E}$ \\
\hline
\end{tabular}


Estas informações podem significar que a população estudada vive comuna grande insegurança financeira e que, por seremidosos, isso agrava o quadro. Parece que uma característica deste estudo é se referir a uma população idosa de baixo poder aquisitivo. Esta condição pode levar as pessoas a se sujeitaremao mercado de trabalho informal ou à dependêndia de parentes, o que semdúvida provoca desgaste entre as relações familiares. Os astos com o adbecer e coma condição crônica toma ainda mais precárias as condições de vida e saúde da população deste estudb ${ }^{10}$.

As condições de facilidades domésticas foram analisadas através da posse de bens de consumo duráveis, identificando que alguns itens são habituais na população e apresentadas na Tabela 4. São eles geladeira (95\%) e televisão a cores (90\%). Otros itens como rádio com $\Phi(55 \%)$, máquina de lavar roupas (52,5\%) e videocassete (25\%) forammenos freqüentes. Oautomóvel foi o bemque registrou menor freqüência (12,5\%) e corrdbora a concepção da árdla tarefa de locomoção destas pessoas.

\section{Tabela 4}

Bens de consumo dos portadores de lesões crônicas -HG B-2005

\begin{tabular}{|c|c|c|}
\hline $\begin{array}{c}\text { Concip̧öes de } \\
\text { mo adid. }\end{array}$ & Vo & $\pi$ \\
\hline Ro & $?$ & 55,0 \\
\hline IV a coes & 36 & 90,0 \\
\hline $\begin{array}{l}\text { Máqu na de } \\
\text { lave ro ips: }\end{array}$ & 21 & 32,5 \\
\hline Gcladcira & 38 & 95,0 \\
\hline YJos & 10 & 25,0 \\
\hline Allominge & 5 & 17.5 \\
\hline
\end{tabular}

Chama a atenção que quatro padientes não têm televisão no domálio. Este eletrodoméstico é uma expressiva altemativa de lazer, informação e conforto para aqueles que não podem sair de seu ambiente cotidiano com freqüência. A TV é sempre uma possibilidade de lazer e distração para as pessoas ${ }^{11}$.

O padrão de vida ea existência de fortes de lazer dentro de casa são aspectos importantes para um entendimento de qualidade de vida, principalmente para pessoas idbsas elou comlesões crônicas e que, por conseguinte, possuem dificuldades de locomoção ${ }^{12}$. Unta moradia adequada, provida de reaursos eletrodomésticos básicos e infra-estrutura sanitánia pode ser relacionada com satisfação, já que foramatendidas as condições níninas materiais para se falar emqualidade de vida?.

\section{CONSIDERAÇÕES FINAIS}

Neste perfil populacional, obsenva-se uma proporção de adultos, idosos e a maioria vivendo sozinhos. A taxa de participação desta população no mercado de trabalho formal é mito pequena, onde tambémse confirma que, à medida que aumenta a idade, dímini a presença no mercado de trabalho. No entanto, é mito provável que os adutos, indusive os idosos, estejamempostos de trabalho informais. De qualquer forma o que chama a atenção, segundo a Tabela 3, é que 13 pacientes (32,5\%) rão possuem renda de qualquer tipo.

Sabese que há una estreita relação entre o estado de saúde, o nível de escolaridade e a participação na força laboral ${ }^{7-8}$. Nesta população, os homens têm escolaridade mais alta que as miheres, e aqueles commais idade têmuma escolaridade pior do que os mais jovens. O padrão de desigualdade educacional pela idade e sexo com certeza influencia também a presença das pessoas na força laboral, e isso, por sua vez, é relevante para influenciar a percepção de qualidade de vida'2 .

A idade é um importante determinante de atoperceppão e saúde As dhances de os idbsos relatarem una saúde negativa são maiores do que autras faixas etárias ${ }^{13-14}$, assimcono ser portador de una lesão cô̂ica já foi referido em outros estudos como um forte influenciador da autoperceppāa de qualidade de vida prinapalmerte emidosos ${ }^{\circ}$.

Segundb estudbs já realizados ${ }^{12-13}$, a variável renda influenda a a toperceepção de qualidade de vida Os idbsos comrenda nais baixa apresentam una percepção nim de qualidade de vica. Por a tro lado, una renda devada é u mindicador robusto de una boa percepsça de qualidade de vida Alguns a tores já nostraramque a percepsão de morbidade pode ser maior entre os estratos sociceconônicos menos favoredidos, o que pode estar reladionadb, dertre autros aspectos, ao valor atribuídb ao corpo, espedialmente tendo emvista a necessidade de trabalhar, possivelmente mas premente entre os grupos de nenor renda ${ }^{912}$.

Existemevidências tambémde que a baixa renda dos idosos atua negativamente no comportamento saudável no anbiente donialiar, no acesso aos senviços de saúde, nos aidados coma saúde e no acesso aos rearsos materiais ${ }^{10 ;}{ }^{15}$. Há evidêndias de que os mais pobres possuambaixa adesão aos tratamentos e têm pouco acesso aos medicamentos, o que se reflete nas condições de saúde do indivídu $0^{6-8}$. 
A população aqui estudada tem como condição commseremportadores de lesões crônicas de pele e referemter vivido as dificuldades de acesso aos senviços de saúde, convivendo com o sofrimento contúnu provocadb pela dor causada pala lesão, assim como coma limitação para deambulação.

Estes aspectos sem dúmida influenciam a participação destes sujeitos na sociedade e quiçá até roodfiquemsuas aspirações na vida. Talvez todas essas questões juntas provoquemnas pessoas estratégias adaptatives de enfrentamento, aja lógica e coerênia são próprias e inegáveis. ${ }^{11}$

Os dados revelamquea população deste estudo temuna condição financeira talvez precária emvirtude de não terem atividade laboral e/ou vínculo empregatício e, aparentemente, sem condições de midanças no estilo de vida e umáralo difíil de ser

\section{Referências}

1KapdanRMQ Qlityy oflife, resarceallocdion andtheUS. Helthcere cisis. In Seid, BMF; ZamonCML Q diidadedevichesaúde aspedtos

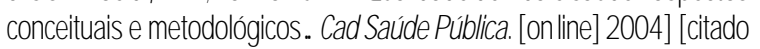

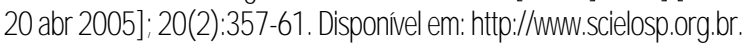

2.Orgarização Mndial da SaúdeOME Giidados inovadores para Condições Crônicas: Componentes estruturais deAcão: Relatónio Mndal/ OrgarizaçãoMndala deSaúdeBraslia, [2003]. Dspponíed enhttprwwrvopess.gov. Acessadbem20 demai 2003.

3.FleckMPA ChadhamovichE, Trentini CAdicação da versão em português doinstrumentoabreviado deavaliação dequalidadede vida 'WHOOQL-Bre'. PevSaúdePúdica2000; 34: 178-83.

4. Gupodeestudosemqualidadedevida (whogd-group). Versão emPortuguês dos Instrumetos deAdiação dequalidadedeVida [1998]. Dsponíve emhlttp//Murufrogs.br. Acessoem 30 set2003.

5. Rouquayrd MZ, AmeicaFilho, N Epidemidogia\&Saúde 6eed Rio deJanero(B): Meds; 2003. 728p.

6-Seid BMF, Zarnon CM Qualidade de vica e saúde aspectos conceituris entodblógicos. CadSaúdePública2004; 20(2): 89-93.

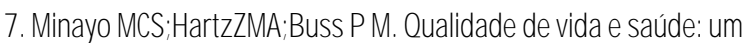
debatenecessário. RevclêndaeSádeColetiva2000; 11(5): 17-18.

8. Buss PM Promoção da saúde equalidadedevida. PevClêndae SaúdeColetiva2000; 1 (2): 145-50.

9. Morgan,GAssesmet of quality of lifein palliativecare Intemetiond J PalliativeNurs2000; 6(8): 406-10.

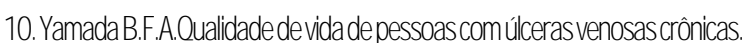

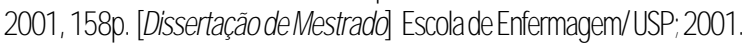

11 Trentini M Silva DGV, MartinsCR:AntorioMC, Tomaz EE, DarteR Qualidade devida dos individuos comdoenças cardovesalares crônicas edabetes mellitus. RevGaúdhaErferm1990; 11(2): 20-7 quebrado, o que sem dúmida pode influenciar a autopercepsão de qualidade de vida'.

Oenfermero, ao identificar as necessidade de sua clientela, reconhecendo as limitações de seus padientes, poderá agregar ao tratamento da lesão estratégias até individualizadas para promover, além do controle da lesão, o conforto, o dívio, a moderação da dor, tudo isto para ajudar a melhorar a qualidade de vica desta dientela

A finalidade do atendimento de umconsultónio de enfermagememhospital público para pacientes com lesões crônicas de pele não é sua a ra terapêtica já que da não é possível para mitas condições crônicas. No entanto, é possível que as intenvenções de enfermagem contribuampara a redução da morbiclade, assimconto para a diminuição do impacto da dbença na qualidade de vica desses indvíduos.

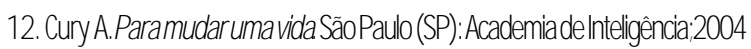

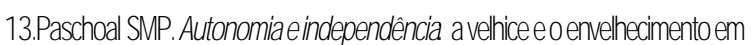
visãogdbalizada Š̃oPalo(\$):Athereu 2002p311-23.

14.VerasRP. Pásjovemcomcabelos brancos: asaúdedoidbsono Brasil. Riodejaneiro(R):: RelumeDmará/l日y; 1994.

15.Farias SNP; Zätoune RCGAinterferência da globalização na qualidadedevicanotrabalho: a percepscão dos trabalhadores de effermagemEscAmaNery RevEfrerm2004,8(3): 386-92

\section{Sobre os Autores}

\section{AraluciaPanos Dos}

Mestrepelo ProgramadeMestradb dafaculdadedeE Efermagenda U甲I, EremeriradbMnistéiodaSaúde

\section{LotlaDopiodastra}

Profa. Oientadora Doutora do Programa de Mestrado em

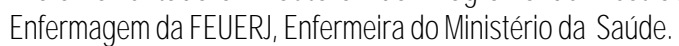
E-nail: dopico@ig.combr 